\title{
Editorial
}

\section{The new kid on the block: the unfolded protein response in the pathogenesis of atherosclerosis}

\author{
M Vasa-Nicotera ${ }^{\star, 1}$ \\ 1 Department of Cardiovascular Sciences, University of Leicester, Glenfield \\ Hospital, Clinical Science Wing, Groby Road, Leicester LE3 9QP, UK \\ * Corresponding author: M Vasa-Nicotera; E-mail: mvn1@le.ac.uk \\ Cell Death and Differentiation (2004) 11, S10-S11. \\ doi:10.1038/sj.cdd. 4401468
}

Atherosclerosis is a complex multifactorial disease, and its clinical manifestations account for the majority of deaths in Western society. The process is believed to initiate at sites of endothelial injury, and it progresses with cholesterol deposition, infiltration of monocytic cells and reactive proliferation of smooth muscle cells within the vessel walls. Macrophages are the most prominent cell type in atherosclerotic lesions. They are present throughout the process of lesion development and characteristically accumulate 'free' cholesterol - an unesterified form of cholesterol. This accumulates in endoplasmic reticular (ER) membranes, which are normally devoid of cholesterol. Such abnormal accumulation of 'free' cholesterol has several adverse effects on ER function, and ultimately results in a stress response known as the unfolded protein response (UPR).

The ER is a primary site for correct protein folding and maturation. ER proteins and several transmembrane proteins, which include signalling and secretory proteins, are normally folded within the ER. A quality-control system ensures that incorrectly folded proteins are degraded before entering their sorting path in the Golgi. Pathological conditions may interfere with correct protein folding and trigger the induction of UPRresponsive genes such as chaperones, proteases and some that regulate protein translation. Although the UPR may be a reactive physiological response to stress, it may also be a prelude to cell death.

In addition to cholesterol overload, other risk factors for atherosclerosis such as hyperhomocysteinaemia can also trigger ER stress and the UPR in endothelial cells, which may contribute to endothelial cell apoptosis. ${ }^{1}$ So, at both ends of the atherosclerotic process - from the initial endothelial cell dysfunction to the final plaque rupture prompted by macrophage cell death - the UPR and ER stress may play important roles.

In this issue of $C D D$, two intriguing reviews examine the role and implications of the UPR in the control of apoptosis and the pathogenesis of atherosclerosis. Austin et al. ${ }^{2}$ describe the role of hyperhomocysteinaemia as a risk factor for atherosclerosis and take a close look at the potential cellular mechanism by which homocysteine regulates endothelial cell function - this includes the activation of the UPR and apoptosis of endothelial cells by hyperhomocysteinaemia. Tabas $^{3}$ focuses on macrophage apoptosis and how it is influenced by the cholesterol-induced UPR. Both articles also describe the role of other important proatherogenic factors such as proinflammatory agents and oxidative/nitrosative stress, as well as the contrasting pro- and antiapoptotic effects of cholesterol in macrophages. For example, the atherogenic effects of hyperhomocysteinaemia have been linked to increased oxygen/nitrogen species and activation of proinflammatory reactions.

So how exactly does homocysteine induce the UPR response and contribute to atherosclerosis? Homocysteine treatment induces ER stress response genes such as GRP78, GRP94, Herp and RTP, and UPR genes such as PERK and IRE-1; however, evidence is not yet available on the relevance of these events to endothelial cell apoptosis in vivo. Homocysteine can trigger other forms of cell death directly. For example, anoikis - cell death induced by detachment from the basement membrane - has been observed after treatment with homocysteine, but this can also induce proapoptotic factors. Therefore, the relative role of these death-inducing pathways still remains to be established.

What is the role of UPR responses in the onset of apoptosis? A lot of attention has recently been directed towards ER-generated signals in the onset of both apoptotic and nonapoptotic cell death. For example, the UPR has been linked to neurodegeneration and autophagocytic cell death of Purkinje cells that is caused by the Lurcher ion channel mutation. ${ }^{4} \mathrm{~A}$ similar type of death may be responsible for neurodegeneration in Caenorhabditis elegans. ${ }^{5}$ The UPR response is a well-defined set of genes and transcriptional factors. In endothelial cells, homocysteine seems to activate IRE-1, which binds to TRAF2, and stress kinases such as JNK; however, little is known about how these signalling pathways are coupled with the activation of apoptotic cell death, and there is no evidence yet that these processes take place in macrophages or endothelial cells during atherogenesis. It is also likely that UPR may lead to other forms of cell death, which may be indistinguishable from apoptosis in vivo. Autophagy, for example, is an active form of cell death in which mitochondria remain intact, and autophagocytic vacuoles are generated within dying cells. Nevertheless, the nucleus also condenses in this form of cell death, and without ultrastructural imaging it would be virtually impossible to discriminate the two forms.

Is accelerated cell death the whole story for the initiation of atherosclerosis? Endothelial cell dysfunction and damage are believed to be the early events that lead to macrophage adhesion to the vessel wall and subsequent plaque formation. Although cell death may be a relevant component of this initial process, there is also substantial evidence for accelerated cell senescence and dysfunctional endothelial cell renewal. Senescent endothelial cells are present in atherosclerotic 
lesions ${ }^{6}$ and homocysteine can accelerate endothelial senescence. $^{7}$

The issue of apoptosis versus other cellular responses to stress becomes even more complex when the macrophage response to stress is examined; oxidized cholesterol may activate the mitochondrial pathway. On the other hand, oxidized LDL may also prevent apoptosis - the outcome might depend on the level of injury and the time during the development of the atherosclerotic insult that the insult occurs.

In conclusion, the reviews presented in this issue of $C D D$ highlight a number of interesting questions. Although the general role of macrophage or metabolic dysfunction has been the subject of widespread discussion, the novel aspect raised by the two reviews is the possibility that a common set of events, namely the activation of ER stress and the UPR, may lead to the demise of different cell types at different disease stages. Do all common risk factors induce such a response? Does the UPR result in apoptosis or in other forms of cell death, which may be insensitive to treatment with caspase inhibitors? These issues still remain open and will require further investigation.

\section{Key points}

- Apoptosis plays a relevant role in atherosclerosis.

- Free cholesterol affects ER function, resulting in a stress response (UPR).

- Hyperhomocysteinaemia, a risk factor for atherosclerosis, also affects UPR.

- Macrophage apoptosis may be caused by the UPR, which is itself caused by cholesterol accumulation.

1. Rao RV et al. (2004) Cell Death Differ. 11: 372-380

2. Austin RC et al. (2004) Cell Death Differ. 11(S1): S56-S64

3. Tabas I (2004) Cell Death Differ. 11(S1): S12-S16

4. Yue $Z$ et al. (2002) Neuron 35: 921-933

5. Driscoll M and Gerstbrein B (2003) Nat. Rev. Genet. 4(3): 181-194

6. Minamino T et al. (2002) Circulation 105: 1541-1544

7. Xu D et al. (2000) FEBS Lett 17; 470(1): 20-24 